\title{
Solutions and Stability of Generalized Mixed Type QC Functional Equations in Random Normed Spaces
}

\author{
Yeol Je Cho, ${ }^{1}$ Madjid Eshaghi Gordji, ${ }^{2}$ and Somaye Zolfaghari ${ }^{3}$ \\ ${ }^{1}$ Department of Mathematics Education and the RINS, Gyeongsang National University, \\ Chinju 660-701, Republic of Korea \\ ${ }^{2}$ Department of Mathematics, Semnan University, P.O. Box 35195-363, Semnan, Iran \\ ${ }^{3}$ Department of Mathematics, Urmia University, Urmia, Iran \\ Correspondence should be addressed to Madjid Eshaghi Gordji, madjid.eshaghi@gmail.com
}

Received 29 July 2010; Accepted 31 August 2010

Academic Editor: Vijay Gupta

Copyright (C) 2010 Yeol Je Cho et al. This is an open access article distributed under the Creative Commons Attribution License, which permits unrestricted use, distribution, and reproduction in any medium, provided the original work is properly cited.

We achieve the general solution and the generalized stability result for the following functional equation in random normed spaces (in the sense of Sherstnev) under arbitrary t-norms: $f(x+$ $k y)+f(x-k y)=k^{2}[f(x+y)+f(x-y)]+\left(2\left(k^{2}-1\right) / k^{2}(k-2)\right) f(k x)-\left(\left(k^{3}-k^{2}-k+1\right) / 2(k-\right.$ 2)) $f(2 x)+\tilde{f}(2 y)-8 \tilde{f}(y)$, where $\tilde{f}(y):=f(y)-f(-y)$ for any fixed integer $k$ with $k \neq 0, \pm 1,2$.

\section{Introduction}

In 1940, the stability problem of functional equations originated from a question given by Ulam [1], that is, the stability of group homomorphisms.

Let $\left(G_{1}, \cdot\right)$ be a group and $\left(G_{2}, *, d\right)$ be a metric group with the metric $d$. For any $\epsilon>0$, does there exist $\delta>0$, such that, if a mapping $h: G_{1} \rightarrow G_{2}$ satisfies inequality $d(h(x \cdot y), h(x) * h(y))<\delta$.

For all $x, y \in G_{1}$, then there exists a homomorphism $H: G_{1} \rightarrow G_{2}$ with $d(h(x), H(x))<\epsilon$ for all $x \in G_{1}$ ?

In other words, under what condition, does there exists a homomorphism near an approximate homomorphism? The concept of stability for functional equation arises when we replace the functional equation by an inequality which acts as a perturbation of the equation.

In 1941, Hyers [2] gave a first affirmative answer to the question of Ulam for Banach spaces. 
Let $f$ be a mapping between Banach spaces $E$ and $E^{\prime}$ such that, for some $\delta>0$,

$$
\|f(x+y)-f(x)-f(y)\| \leq \delta, \quad \forall x, y \in E
$$

Then there exists a unique additive mapping $T: E \rightarrow E^{\prime}$ such that

$$
\|f(x)-T(x)\| \leq \delta, \quad \forall x, y \in E
$$

Moreover, if $f(t x)$ is continuous in $t \in \mathbb{R}$ for any fixed $x \in E$, then $T$ is linear.

In 1978, Rassias [3] provided a generalization of Hyers' Theorem which allows the Cauchy difference to be unbounded. In 1991, Gajda [4] answered the question for the case $p>1$, which was raised by Rassias. This new concept is known as the Hyers-Ulam-Rassias stability of functional equations (see [5-17]).

The functional equation

$$
f(x+y)+f(x-y)=2 f(x)+2 f(y)
$$

is related to symmetric biadditive mapping. It is natural that this equation is called a quadratic functional equation. In particular, every solution of the quadratic equation (1.3) is said to be a quadratic mapping. It is well known that a mapping $f$ between real vector spaces $E$ and $E^{\prime}$ is quadratic if and only if there exits a unique symmetric biadditive mapping $B$ such that $f(x)=B(x, x)$ for all $x \in E$ (see $[5,18])$. The biadditive mapping $B$ is given by

$$
B(x, y)=\frac{1}{4}(f(x+y)-f(x-y)), \quad \forall x, y \in E .
$$

Hyers-Ulam-Rassias stability problem for the quadratic functional equation (1.3) was proved by Skof [19] for a mapping $f: E \rightarrow E^{\prime}$, where $E$ is a normed space and $E^{\prime}$ is a Banach space. Cholewa [20] noticed that the theorem of Skof is still true if the relevant domain $A$ is replaced by an abelian group. In [21], Czerwik proved the Hyers-Ulam-Rassias stability of (1.3) and Grabiec [22] generalized these results mentioned above.

Recently, Jun and Kim [23] introduced the following cubic functional equation:

$$
f(2 x+y)+f(2 x-y)=2 f(x+y)+2 f(x-y)+12 f(x), \quad \forall x, y \in X,
$$

where $f$ is a mapping from a real vector space $X$ into a real vector space $Y$ and they established the general solution and the generalized Hyers-Ulam-Rassias stability for the functional equation (1.5). The function $f(x)=x^{3}$ satisfies the functional equation (1.5), which is thus called a cubic functional equation. Every solution of the cubic functional equation is said to be a cubic function. Also, Jun and Kim proved that a function $f$ between real vector spaces $X$ and $Y$ is a solution of (1.5) if and only if there exits a unique function $C: X \times X \times X \rightarrow Y$ such that $f(x)=C(x, x, x)$ for all $x \in X$ and $C$ is symmetric for each fixed one variable and is additive for fixed two variables.

In the sequel, we adopt the usual terminology, notations and conventions of the theory of random normed spaces as in [24-28]. Throughout this paper, $\Delta^{+}$is the space of distribution 
functions, that is, the space of all mappings $F: \mathbb{R} \cup\{-\infty, \infty\} \rightarrow[0,1]$ such that $F$ is leftcontinuous and nondecreasing on $\mathbb{R}, \mathbb{F}(0)=0$ and $F(+\infty)=1$. $D^{+}$is a subset of $\Delta^{+}$consisting of all functions $F \in \Delta^{+}$for which $l^{-} F(+\infty)=1$, where $l^{-} f(x)$ denotes the left limit of the function $f$ at the point $x$, that is, $l^{-} f(x)=\lim _{t \rightarrow x^{-}} f(t)$. The space $\Delta^{+}$is partially ordered by the usual pointwise ordering of functions, that is, $F \leq G$ if and only if $F(t) \leq G(t)$ for all $t \in \mathbb{R}$. The maximal element for $\Delta^{+}$in this order is the distribution function $\varepsilon_{0}$ given by

$$
\varepsilon_{0}(t)= \begin{cases}0, & \text { if } t \leq 0 \\ 1, & \text { if } t>0\end{cases}
$$

Definition 1.1 (see [27]). A mapping $T:[0,1] \times[0,1] \rightarrow[0,1]$ is called a continuous triangular norm (briefly, a continuous $t$-norm) if $T$ satisfies the following conditions:

(a) $T$ is commutative and associative;

(b) $T$ is continuous;

(c) $T(a, 1)=a$ for all $a \in[0,1]$;

(d) $T(a, b) \leq T(c, d)$ whenever $a \leq c$ and $b \leq d$ for all $a, b, c, d \in[0,1]$.

Typical examples of continuous $t$-norms are $T_{P}(a, b)=a b, T_{M}(a, b)=\min (a, b)$ and $T_{L}(a, b)=\max (a+b-1,0)$ (the Lukasiewicz $t$-norm).

Recall (see $[29,30]$ ) that, if $T$ is a $t$-norm and $\left\{x_{n}\right\}$ is a given sequence of numbers in $[0,1]$, then $T_{i=1}^{n} x_{i}$ is defined recurrently by

$$
T_{i=1}^{1} x_{i}=x_{1}, \quad T_{i=1}^{n} x_{i}=T\left(T_{i=1}^{n-1} x_{i}, x_{n}\right), \quad \forall n \geq 2,
$$

and $T_{i=n}^{\infty} x_{i}$ is defined as $T_{i=1}^{\infty} x_{n+i}$.

It is known [30] that, for the Lukasiewicz $t$-norm, the following implication holds:

$$
\lim _{n \rightarrow \infty}\left(T_{L}\right)_{i=1}^{\infty} x_{n+i}=1 \Longleftrightarrow \sum_{n=1}^{\infty}\left(1-x_{n}\right)<\infty
$$

Definition 1.2 (see [28]). A random normed space (briefly, $R N$-space) is a triple $(X, \mu, T)$, where $X$ is a vector space, $T$ is a continuous $t$-norm and $\mu$ is a mapping from $X$ into $D^{+}$satisfying the following conditions:

$(R N 1) \mu_{x}(t)=\varepsilon_{0}(t)$ for all $t>0$ if and only if $x=0$;

(RN2) $\mu_{\alpha x}(t)=\mu_{x}(t /|\alpha|)$ for all $x \in X$, and $\alpha \in \mathbb{R}$ with $\alpha \neq 0$;

(RN3) $\mu_{x+y}(t+s) \geq T\left(\mu_{x}(t), \mu_{y}(s)\right)$ for all $x, y \in X$ and $t, s \geq 0$.

Every normed spaces $(X,\|\cdot\|)$ defines a random normed space $\left(X, \mu, T_{M}\right)$, where

$$
\mu_{x}(t)=\frac{t}{t+\|x\|}, \quad \forall t>0
$$

and $T_{M}$ is the minimum $t$-norm. This space is called the induced random normed space. 
Definition 1.3. Let $(X, \mu, T)$ be a $\mathrm{RN}$-space.

(1) A sequence $\left\{x_{n}\right\}$ in $X$ is said to be convergent to a point $x \in X$ if, for any $\epsilon>0$ and $\lambda>0$, there exists a positive integer $N$ such that $\mu_{x_{n}-x}(\epsilon)>1-\lambda$ for all $n \geq N$.

(2) A sequence $\left\{x_{n}\right\}$ in $X$ is called a Cauchy sequence if, for any $\epsilon>0$ and $\lambda>0$, there exists a positive integer $N$ such that $\mu_{x_{n}-x_{m}}(\epsilon)>1-\lambda$ for all $n \geq m \geq N$.

(3) A RN-space $(X, \mu, T)$ is said to be complete if every Cauchy sequence in $X$ is convergent to a point in $X$.

Theorem 1.4 (see [27]). If $(X, \mu, T)$ is a RN-space and $\left\{x_{n}\right\}$ is a sequence in $X$ such that $x_{n} \rightarrow x$, then $\lim _{n \rightarrow \infty} \mu_{x_{n}}(t)=\mu_{x}(t)$ almost everywhere.

The stability of different functional equations in fuzzy normed spaces and random normed spaces has been studied in [13, 31-42]. $k \neq 0, \pm 1,2$ :

In this paper, we deal with the following functional equation for fixed integers $k$ with

$$
\begin{aligned}
f(x+k y)+f(x-k y)= & k^{2}[f(x+y)+f(x-y)]+\frac{2\left(k^{2}-1\right)}{k^{2}(k-2)} f(k x) \\
& -\frac{k^{3}-k^{2}-k+1}{2(k-2)} f(2 x)+\tilde{f}(2 y)-8 \tilde{f}(y)
\end{aligned}
$$

where $\tilde{f}(y):=f(y)-f(-y)$ on random normed spaces. It is easy to see that the mapping $f(x)=a x^{2}+b x^{3}+c$ is a solution of the functional equation (1.10). In Section 2, we investigate the general solution of functional equation (1.10) when $f$ is a mapping between vector spaces and, in Section 3, we establish the stability of the functional equation (1.10) in RN-spaces.

\section{General Solutions}

Before proceeding to the proof of Theorem 2.3 which is the main result in this section, we need the following two lemmas.

Lemma 2.1. If an even function $f: X \rightarrow Y$ with $f(0)=0$ satisfies (1.10), then $f$ is quadratic.

Proof. Setting $x=0$ in (1.10), by the evenness of $f$, we obtain

$$
f(k y)=k^{2} f(y), \quad \forall y \in X .
$$

Interchanging $x$ with $y$ in (2.1), we have

$$
f(k x)=k^{2} f(x), \quad \forall x \in X
$$

Letting $y=0$ in (1.10), we have

$$
f(x)=\frac{-1}{k^{2}(k-2)} f(k x)+\frac{k-1}{4(k-2)} f(2 x), \quad \forall x \in X .
$$


It follows from (2.2) and (2.3) that

$$
f(2 x)=4 f(x), \quad \forall x \in X
$$

According to (2.2), (2.4) and using the evenness of $f,(1.10)$ can be written as

$$
f(x+k y)+f(x-k y)=k^{2} f(x+y)+k^{2} f(x-y)+2\left(1-k^{2}\right) f(x), \quad \forall x, y \in X .
$$

Replacing $x$ by $k x$ in (2.5) and then using (2.2), we obtain

$$
f(k x+y)+f(k x-y)=f(x+y)+f(x-y)+2\left(k^{2}-1\right) f(x), \quad \forall x, y \in X
$$

Interchanging $x$ with $y$ in (2.5), by the evenness of $f$, we have

$$
f(k x+y)+f(k x-y)=k^{2} f(x+y)+k^{2} f(x-y)+2\left(1-k^{2}\right) f(y), \quad \forall x, y \in X
$$

But, since $k \neq 0, \pm 1,2$, it follows from (2.6) and (2.7) that

$$
f(x+y)+f(x-y)=2 f(x)+2 f(y), \quad \forall x, y \in X,
$$

which shows that $f$ is quadratic. This completes the proof.

Lemma 2.2. If an odd function $f: X \rightarrow Y$ satisfies (1.10), then $f$ is cubic.

Proof. Letting $x=0$ in (1.10), by the oddness of $f$, we have

$$
f(2 y)=8 f(y), \quad \forall y \in X
$$

Setting $y=0$ in (1.10) and then using (2.9), we obtain

$$
f(k x)=k^{3} f(x), \quad \forall x \in X
$$

According to (2.9), (2.10) and using the oddness of $f,(1.10)$ can be written as

$$
f(x+k y)+f(x-k y)=k^{2} f(x+y)+k^{2} f(x-y)+2\left(1-k^{2}\right) f(x), \quad \forall x, y \in X
$$

Letting $y=x$ in (2.11) and using (2.9), by the oddness of $f$, it follows that

$$
f((k+1) x)=f((k-1) x)+2\left(1+3 k^{2}\right) f(x), \quad \forall x \in X
$$


Replacing $x$ by $(k-1) x$ in $(2.11)$, we have

$$
\begin{aligned}
f((k-1) x+k y)+f((k-1) x-k y) & \\
= & k^{2} f((k-1) x+y)+k^{2} f((k-1) x-y) \\
& +2\left(1-k^{2}\right) f((k-1) x), \quad \forall x, y \in X .
\end{aligned}
$$

Now, replacing $x$ by $(k+1) x$ in (2.11) and using (2.12), we have

$$
\begin{aligned}
f((k+1) x+k y)+f((k+1) x-k y) & \\
= & k^{2} f((k+1) x+y)+k^{2} f((k+1) x-y) \\
& +2\left(1-k^{2}\right) f((k-1) x)+4\left(1-k^{2}\right)\left(3 k^{2}+1\right) f(x), \quad \forall x, y \in X .
\end{aligned}
$$

Substituting $x+y$ with $x$ in (2.11) and then $x-y$ with $x$ in (2.11), we obtain

$$
\begin{aligned}
\begin{aligned}
f(x+ & (k+1) y)+f(x-(k-1) y) \\
& =k^{2} f(x+2 y)+2\left(1-k^{2}\right) f(x+y)+k^{2} f(x), \quad \forall x, y \in X,
\end{aligned} \\
\begin{aligned}
f(x- & (k+1) y)+f(x+(k-1) y) \\
& =k^{2} f(x-2 y)+2\left(1-k^{2}\right) f(x-y)+k^{2} f(x), \quad \forall x, y \in X .
\end{aligned}
\end{aligned}
$$

If we subtract (2.16) from (2.15), we have

$$
\begin{aligned}
f(x+ & (k+1) y)-f(x-(k+1) y) \\
= & k^{2} f(x+2 y)-k^{2} f(x-2 y)+f(x+(k-1) y)-f(x-(k-1) y) \\
& +2\left(1-k^{2}\right) f(x+y)-2\left(1-k^{2}\right) f(x-y), \quad \forall x, y \in X .
\end{aligned}
$$

Interchanging $x$ with $y$ in (2.17) and using the oddness of $f$, we get

$$
\begin{aligned}
f((k+1) x+y)+f((k+1) x-y) & \\
= & k^{2} f(2 x+y)+k^{2} f(2 x-y)+f((k-1) x+y)+f((k-1) x-y) \\
& +2\left(1-k^{2}\right) f(x+y)+2\left(1-k^{2}\right) f(x-y), \quad \forall x, y \in X .
\end{aligned}
$$


Thus it follows from (2.14) and (2.18) that

$$
\begin{aligned}
& f((k+1) x+k y)+f((k+1) x-k y) \\
&=k^{2} f((k-1) x+y)+k^{2} f((k-1) x-y)+k^{4} f(2 x+y)+k^{4} f(2 x-y) \\
&+2 k^{2}\left(1-k^{2}\right) f(x+y)+2 k^{2}\left(1-k^{2}\right) f(x-y) \\
&+2\left(1-k^{2}\right) f((k-1) x)+4\left(1-k^{2}\right)\left(3 k^{2}+1\right) f(x), \quad \forall x, y \in X .
\end{aligned}
$$

Again, substituting $x+y$ with $y$ in (2.11) and then $x-y$ with $y$ in (2.11), we get, by the oddness of $f$,

$$
\begin{aligned}
& \begin{array}{l}
f((k+1) x+k y)-f((k-1) x+k y) \\
\quad=k^{2} f(2 x+y)+k^{2} f(-y)+2\left(1-k^{2}\right) f(x), \quad \forall x, y \in X, \\
f((k+1) x-k y)-f((k-1) x-k y) \\
\quad=k^{2} f(2 x-y)+k^{2} f(y)+2\left(1-k^{2}\right) f(x), \quad \forall x, y \in X .
\end{array}
\end{aligned}
$$

Then, by adding (2.20) to (2.21) and then using (2.13), we have

$$
\begin{aligned}
f((k+1) x+k y)+f((k+1) x-k y) & \\
= & k^{2} f((k-1) x+y)+k^{2} f((k-1) x-y)+2\left(1-k^{2}\right) f((k-1) x) \\
& +k^{2} f(2 x+y)+k^{2} f(2 x-y)+4\left(1-k^{2}\right) f(x), \quad \forall x, y \in X .
\end{aligned}
$$

Finally, if we compare (2.19) with (2.22), then we conclude that

$$
f(2 x+y)+f(2 x-y)=2 f(x+y)+2 f(x-y)+12 f(x), \quad \forall x, y \in X
$$

Therefore, $f$ is a cubic function. This completes the proof.

Theorem 2.3. A function $f: X \rightarrow Y$ with $f(0)=0$ satisfies (1.10) for all $x, y \in X$ if and only if there exist functions $Q: X \times X \rightarrow Y$ and $C: X \times X \times X \rightarrow Y$ such that $f(x)=Q(x, x)+C(x, x, x)$ for all $x \in X$, where the function $C$ is symmetric for each fixed one variable and is additive for fixed two variables and $Q$ is symmetric biadditive.

Proof. Let $f$ be a mapping with $f(0)=0$ satisfies (1.10). We decompose $f$ into the even part and odd part by putting

$$
f_{e}(x)=\frac{1}{2}(f(x)+f(-x)), \quad f_{o}(x)=\frac{1}{2}(f(x)-f(-x)), \quad \forall x \in X
$$


It is clear that $f(x)=f_{e}(x)+f_{o}(x)$ for all $x \in X$ and it is easy to show that the functions $f_{e}$ and $f_{o}$ satisfy (1.10). Hence, by Lemmas 2.1 and 2.2, we know that the functions $f_{e}$ and $f_{o}$ are quadratic and cubic, respectively. Thus there exist a symmetric biadditive function $Q: X \times X \rightarrow Y$ such that $f_{e}(x)=Q(x, x)$ for all $x \in X$ and the function $C: X \times X \times X \rightarrow Y$ such that $f_{o}(x)=C(x, x, x)$ for all $x \in X$, where the function $C$ is symmetric for each fixed one variable and is additive for fixed two variables. Therefore, we get $f(x)=C(x, x, x)+Q(x, x)$ for all $x \in X$.

Conversely, let $f(x)=C(x, x, x)+Q(x, x)$ for all $x \in X$, where the function $C$ is symmetric for each fixed one variable and is additive for fixed two variables and $Q$ is biadditive. By a simple computation, one can show that the functions $x \mapsto C(x, x, x)$ and $x \mapsto Q(x, x)$ satisfy the functional equation (1.10). Thus the function $f$ satisfies (1.10). This completes the proof.

\section{Stability Problems}

From now on, we suppose that $X$ is a real linear space, $(Y, \mu, T)$ is a complete $R N$-space and $f: X \rightarrow Y$ is a function with $f(0)=0$ for which there exists a mapping $\rho: X \times X \rightarrow D^{+}$ $\left(\rho(x, y)\right.$ is denoted by $\left.\rho_{x, y}\right)$ with the property:

$$
\begin{aligned}
& \mu_{f(x+k y)+f(x-k y)-k^{2}[f(x+y)+f(x-y)]-\left(2\left(k^{2}-1\right) / k^{2}(k-2)\right) f(k x)+\left(\left(k^{3}-k^{2}-k+1\right) / 2(k-2)\right) f(2 x)-\tilde{f}(2 y)+8 \tilde{f}(y)}(t) \\
& \geq \rho_{x, y}(t), \quad \forall x, y \in X, t>0,
\end{aligned}
$$

where $\tilde{f}(y):=f(y)-f(-y)$ for all $y \in X$.

Theorem 3.1. Let

$$
\begin{aligned}
\lim _{m \rightarrow \infty} T_{j=1}^{\infty}\left(\rho_{0, k^{m+j-1} x}\left(k^{2 m+j} t\right)\right) & =1 \\
& =\lim _{m \rightarrow \infty} \rho_{k^{m} x, k^{m} y}\left(k^{2 m} t\right), \quad \forall x, y \in X, t>0 .
\end{aligned}
$$

Suppose that an even function $f: X \rightarrow Y$ with $f(0)=0$ satisfies inequality

$$
\begin{aligned}
& \mu_{f(x+k y)+f(x-k y)-k^{2}[f(x+y)+f(x-y)]-\left(2\left(k^{2}-1\right) / k^{2}(k-2)\right) f(k x)+\left(\left(k^{3}-k^{2}-k+1\right) / 2(k-2)\right) f(2 x)-\tilde{f}(2 y)+8 \tilde{f}(y)}(t) \\
& \quad \geq \rho_{x, y}(t), \quad \forall x, y \in X, t>0
\end{aligned}
$$

where $\tilde{f}(y):=f(y)-f(-y)$ for all $y \in X$. Then there exists a unique quadratic mapping $Q: X \rightarrow Y$ such that

$$
\mu_{Q(x)-f(x)}(t) \geq T_{j=1}^{\infty}\left(\rho_{0, k^{j-1} x}\left(k^{j} t\right)\right), \quad \forall x \in X, t>0 .
$$


Proof. It follows from (3.3) and the evenness of $f$ that

$$
\begin{aligned}
& \mu_{f(x+k y)+f(x-k y)-k^{2}[f(x+y)+f(x-y)]-\left(2\left(k^{2}-1\right) / k^{2}(k-2)\right) f(k x)+\left(\left(k^{3}-k^{2}-k+1\right) / 2(k-2)\right) f(2 x)}(t) \\
& \geq \rho_{x, y}(t), \quad \forall x, y \in X, t>0 .
\end{aligned}
$$

Setting $x=0$ in (3.5), we get

$$
\mu_{2 f(k y)-2 k^{2} f(y)}(t) \geq \rho_{0, y}(t), \quad \forall y \in X, t>0 .
$$

If we replace $y$ by $x$ in (3.6) and divide both sides of (3.6) by 2, we get

$$
\mu_{f(k x)-k^{2} f(x)}(t) \geq \rho_{0, x}(2 t) \geq \rho_{0, x}(t), \quad \forall x \in X, t>0 .
$$

In other words, we have

$$
\mu_{f(k x) / k^{2}-f(x)}(t) \geq \rho_{0, x}\left(k^{2} t\right), \quad \forall x \in X, t>0 .
$$

Therefore, it follows that

$$
\mu_{f\left(k^{n+1} x\right) / k^{2(n+1)}-f\left(k^{n} x\right) / k^{2 n}}\left(\frac{t}{k^{2 n}}\right) \geq \rho_{0, k^{n} x}\left(k^{2} t\right), \quad \forall x \in X, t>0, n \in \mathbb{N} .
$$

Hence we have

$$
\mu_{f\left(k^{n+1} x\right) / k^{2(n+1)}-f\left(k^{n} x\right) / k^{2 n}}(t) \geq \rho_{0, k^{n} x}\left(k^{2(n+1)} t\right), \quad \forall x \in X, t>0, n \in \mathbb{N} .
$$

This means that

$$
\mu_{f\left(k^{n+1} x\right) / k^{2(n+1)}-f\left(k^{n} x\right) / k^{2 n}}\left(\frac{t}{k^{n+1}}\right) \geq \rho_{0, k^{n} x}\left(k^{(n+1)} t\right), \quad \forall x \in X, t>0, n \in \mathbb{N} .
$$

Since $1>1 / 2+1 / 2^{2}+\cdots+1 / 2^{m}$, by the triangle inequality, it follows that

$$
\begin{aligned}
\mu_{\left(f\left(k^{m} x\right) / k^{2 m}\right)-f(x)}(t) & \geq T_{n=0}^{m-1}\left(\mu_{f\left(k^{n+1} x\right) / k^{2(n+1)}-f\left(k^{n} x\right) / k^{2 n}}\left(\sum_{n=0}^{m-1} \frac{t}{k^{n+1}}\right)\right) \\
& \geq T_{n=0}^{m-1}\left(\rho_{0, k^{n} x}\left(k^{(n+1)} t\right)\right) \\
& =T_{j=1}^{m}\left(\rho_{0, k^{j-1} x}\left(k^{j} t\right)\right), \quad \forall x \in X, t>0 .
\end{aligned}
$$

In order to prove the convergence of the sequence $\left\{f\left(k^{m} x\right) / k^{2 m}\right\}$, we replace $x$ with $k^{m^{\prime}} x$ in (3.12) to find that

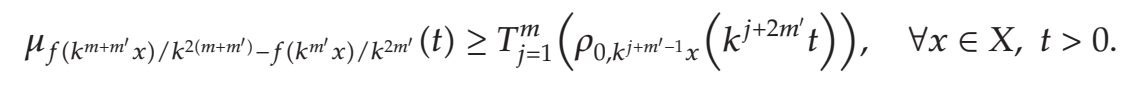


Since the right hand side of inequality (3.13) tends to 1 as $m^{\prime}, m \rightarrow \infty$, the sequence $\left\{f\left(k^{m} x\right) / k^{2 m}\right\}$ is a Cauchy sequence. Therefore, we may define

$$
Q(x)=\lim _{m \rightarrow \infty} \frac{f\left(k^{m} x\right)}{k^{2 m}}, \quad \forall x \in X
$$

Now, we show that $Q$ is a quadratic mapping. In fact, replacing $x, y$ with $k^{m} x$ and $k^{m} y$, respectively, in (3.3) and then taking the limit as $m \rightarrow \infty$, we find that $Q$ satisfies (1.10) for all $x, y \in X$. Therefore, the mapping $Q: X \rightarrow Y$ is quadratic.

To prove (3.4), taking the limit as $m \rightarrow \infty$ in (3.12), we get (3.4).

Finally, to prove the uniqueness of the quadratic function $Q$ subject to (3.4), assume that there exists a quadratic function $Q^{\prime}$ which satisfies (3.4). Since $Q\left(k^{m} x\right)=k^{2 m} Q(x)$ and $Q^{\prime}\left(k^{m} x\right)=k^{2 m} Q^{\prime}(x)$ for all $x \in X$ and $m \in \mathbb{N}$, it follows from (3.4) that

$$
\begin{aligned}
\mu_{Q(x)-Q^{\prime}(x)}(t) & =\mu_{Q\left(k^{m} x\right)-Q^{\prime}\left(k^{m} x\right)}\left(k^{2 m} t\right) \\
& \geq T\left(\mu_{Q\left(k^{m} x\right)-f\left(k^{m} x\right)}\left(k^{2 m-1} t\right), \mu_{f\left(k^{m} x\right)-Q^{\prime}\left(k^{m} x\right)}\left(k^{2 m-1} t\right)\right) \\
& \geq T\left(T_{j=1}^{\infty}\left(\rho_{0, k^{j+m-1} x}\left(k^{2 m+j} t\right)\right), T_{j=1}^{\infty}\left(\rho_{0, k^{j+m-1} x}\left(k^{2 m+j} t\right)\right)\right)
\end{aligned}
$$

for all $x \in X$ and $t>0$. Therefore, by letting $m \rightarrow \infty$ in inequality (3.15), we find that $Q=Q^{\prime}$. This completes the proof.

Theorem 3.2. Let $f: X \rightarrow Y$ be an odd mapping with $f(0)=0$ satisfies inequality

$$
\begin{aligned}
& \mu_{f(x+k y)+f(x-k y)-k^{2}[f(x+y)+f(x-y)]-\left(2\left(k^{2}-1\right) / k^{2}(k-2)\right) f(k x)+\left(\left(k^{3}-k^{2}-k+1\right) / 2(k-2)\right) f(2 x)-\tilde{f}(2 y)+8 \tilde{f}(y)}(t) \\
& \geq \rho_{x, y}(t), \quad \forall x, y \in X, t>0
\end{aligned}
$$

where $\tilde{f}(y):=f(y)-f(-y)$ for all $y \in X$. If

$$
\begin{gathered}
\lim _{m \rightarrow \infty} T_{j=1}^{\infty}\left(T\left(\rho_{0, k^{m+j-1} x}\left(\frac{2 k^{2 j+3 m}}{k^{2}(k-1)} t\right), \rho_{k^{m+j-1} x, 0}\left(\frac{k^{(3 m+2 j)}\left(1-k^{2}\right)}{k^{2}(k-2)} t\right)\right)\right)=1, \\
\lim _{m \rightarrow \infty} \rho_{k^{m} x, k^{m} y}\left(k^{3 m} t\right)=1, \quad \forall x, y \in X, t>0,
\end{gathered}
$$

then there exists a unique cubic mapping $C: X \rightarrow Y$ such that

$$
\mu_{C(x)-f(x)}(t) \geq T_{j=1}^{\infty}\left(T\left(\rho_{0, k^{j-1} x}\left(\frac{2 k^{2 j}}{k^{2}(k-1)} t\right), \rho_{k^{j-1} x, 0}\left(\frac{k^{2 j}\left(1-k^{2}\right)}{k^{2}(k-2)} t\right)\right)\right)
$$

for all $x \in X$ and $t>0$. 
Proof. It follows from (3.15) and the oddness of $f$ that

$$
\begin{aligned}
& \mu_{f(x+k y)+f(x-k y)-k^{2}[f(x+y)+f(x-y)]-\left(2\left(k^{2}-1\right) / k^{2}(k-2)\right) f(k x)+\left(\left(k^{3}-k^{2}-k+1\right) / 2(k-2)\right) f(2 x)-2 f(2 y)+16 f(y)}(t) \\
& \geq \rho_{x, y}(t), \quad \forall x, y \in X, t>0 .
\end{aligned}
$$

By putting $x=0$ in (3.20) and using (RN2), we obtain

$$
\mu_{2 f(2 y)-16 f(y)}(t) \geq \rho_{0, y}(t), \quad \forall y \in X, t>0 .
$$

If we replace $y$ by $x$ in (3.21) and divide both sides of (3.21) by 2, we get

$$
\mu_{f(2 x)-8 f(x)}(t) \geq \rho_{0, x}(2 t) \geq \rho_{0, x}(t), \quad \forall x \in X, t>0 .
$$

Letting $y=0$ in (3.20), we get

$$
\begin{aligned}
& \mu_{2\left(1-k^{2}\right) f(x)-\left(2\left(k^{2}-1\right) / k^{2}(k-2)\right) f(k x)+\left(\left(k^{3}-k^{2}-k+1\right) / 2(k-2)\right) f(2 x)}(t) \\
& \geq \rho_{x, 0}(t), \quad \forall x \in X, \quad t>0 .
\end{aligned}
$$

Therefore, we have

$$
\mu_{k^{2}(k-2) f(x)+f(k x)-\left(k^{2}(k-1) / 4\right) f(2 x)}(t) \geq \rho_{x, 0}\left(\frac{2\left(1-k^{2}\right)}{k^{2}(k-2)} t\right), \quad \forall x \in X, t>0 .
$$

It follows from (3.22) and (3.24) that

$$
\begin{array}{r}
\mu_{f(k x)-k^{3} f(x)}(t) \geq T\left(\rho_{0, x}\left(\frac{2}{k^{2}(k-1)} t\right), \rho_{x, 0}\left(\frac{\left(1-k^{2}\right)}{k^{2}(k-2)} t\right)\right) \\
\forall x \in X, t>0, k \in \mathbb{N} .
\end{array}
$$

Let

$$
\psi(x, x)(t)=T\left(\rho_{0, x}\left(\frac{2}{k^{2}(k-1)} t\right), \rho_{x, 0}\left(\frac{\left(1-k^{2}\right)}{k^{2}(k-2)} t\right)\right), \quad \forall x \in X, t>0
$$

Then we get

$$
\mu_{f(k x)-k^{3} f(x)}(t) \geq \psi_{(x, x)}(t), \quad \forall x \in X, t>0 .
$$

It follows that

$$
\mu_{f(k x) / k^{3}-f(x)}(t) \geq \psi_{(x, x)}\left(k^{3} t\right), \quad \forall x \in X, t>0
$$


Hence we have

$$
\mu_{f\left(k^{n+1} x\right) / k^{3(n+1)}-f\left(k^{n} x\right) / k^{3 n}}\left(\frac{t}{k^{3 n}}\right) \geq \psi_{\left(k^{n} x, k^{n} x\right)}\left(k^{3} t\right), \quad \forall x \in X, t>0,
$$

which implies that

$$
\mu_{f\left(k^{n+1} x\right) / k^{3(n+1)}-f\left(k^{n} x\right) / k^{3 n}}(t) \geq \psi_{\left(k^{n} x, k^{n} x\right)}\left(k^{3(n+1)} t\right), \quad \forall x \in X, t>0, n \in \mathbb{N} .
$$

Thus we have

$$
\mu_{f\left(k^{n+1} x\right) / k^{3(n+1)}-f\left(k^{n} x\right) / k^{3 n}}\left(\frac{t}{k^{n+1}}\right) \geq \psi_{\left(k^{n} x, k^{n} x\right)}\left(k^{2(n+1)} t\right), \quad \forall x \in X, t>0, n \in \mathbb{N} .
$$

Since $1>1 / 2+1 / 2^{2}+\cdots+1 / 2^{m}$, by the triangle inequality, it follows that

$$
\begin{aligned}
\mu_{f\left(k^{m} x\right) / k^{3 m}-f(x)}(t) & \geq T_{n=0}^{m-1}\left(\mu_{f\left(k^{n+1} x\right) / k^{3(n+1)}-f\left(k^{n} x\right) / k^{3 n}}\left(\sum_{n=0}^{m-1} \frac{t}{k^{n+1}}\right)\right) \\
& \geq T_{n=0}^{m-1}\left(\psi_{k^{n} x, k^{n} x}\left(k^{2(n+1)} t\right)\right) \\
& =T_{j=1}^{m}\left(\psi_{k^{j-1} x, k^{j-1} x}\left(k^{2 j} t\right)\right), \quad \forall x \in X, \quad t>0 .
\end{aligned}
$$

In order to prove the convergence of the sequence $\left\{f\left(k^{m} x\right) / k^{3 m}\right\}$, we replace $x$ with $k^{m^{\prime}} x$ in (3.32) to find that

$$
\mu_{f\left(k^{m+m^{\prime}} x\right) / k^{3\left(m+m^{\prime}\right)}-f\left(k^{m^{\prime}} x\right) / k^{3 m^{\prime}}}(t) \geq T_{j=1}^{m}\left(\psi_{k^{j+m^{\prime}-1} x, k^{j+m^{\prime}-1} x}\left(k^{2 j+3 m^{\prime}} t\right)\right) .
$$

Since the right hand side of inequality (3.33) tends to 1 as $m^{\prime}, m \rightarrow \infty$, the sequence $\left\{f\left(k^{m} x\right) / k^{3 m}\right\}$ is a Cauchy sequence. Therefore, we can define

$$
C(x)=\lim _{m \rightarrow \infty} \frac{f\left(k^{m} x\right)}{k^{3 m}}, \quad \forall x \in X
$$

Now, we show that $C$ is a cubic mapping. In fact, replacing $x, y$ with $k^{m} x$ and $k^{m} y$ in (3.15), respectively, and then taking the limit $m \rightarrow \infty$, we find that $C$ satisfies (1.10) for all $x, y \in X$. Therefore, the mapping $C: X \rightarrow Y$ is cubic.

Letting the limit $m \rightarrow \infty$ in (3.32), we get inequality (3.18) by (3.26). 
Finally, to prove the uniqueness of the cubic function $C$ subject to inequality (3.19), assume that there exists a cubic function $C^{\prime}$ which satisfies inequality (3.19). Since $C\left(k^{m} x\right)=$ $k^{3 m} C(x)$ and $C^{\prime}\left(k^{m} x\right)=k^{3 m} C^{\prime}(x)$ for all $x \in X$ and $m \in \mathbb{N}$, it follows from (3.19) that

$$
\begin{aligned}
& \mu_{C(x)-C^{\prime}(x)}(t) \\
& =\mu_{C\left(k^{m} x\right)-C^{\prime}\left(k^{m} x\right)}\left(k^{3 m} t\right) \geq T\left(\mu_{C\left(k^{m} x\right)-f\left(k^{m} x\right)}\left(k^{3 m-1} t\right), \mu_{f\left(k^{m} x\right)-C^{\prime}\left(k^{m} x\right)}\left(k^{3 m-1} t\right)\right) \\
& \geq T\left[T_{j=1}^{\infty}\left(T\left(\rho_{0, k^{m+j-1} x}\left(\frac{2 k^{2 j+3 m}}{k^{2}(k-1)} t\right), \rho_{k^{m+j-1} x, 0}\left(\frac{k^{(3 m+2 j)}\left(1-k^{2}\right)}{k^{2}(k-2)} t\right)\right)\right),\right. \\
& \left.\quad T_{j=1}^{\infty}\left(T\left(\rho_{0, k^{m+j-1} x}\left(\frac{2 k^{2 j+3 m}}{k^{2}(k-1)} t\right), \rho_{k^{m+j-1} x, 0}\left(\frac{k^{(3 m+2 j)}\left(1-k^{2}\right)}{k^{2}(k-2)} t\right)\right)\right)\right]
\end{aligned}
$$

for all $x \in X$ and $t>0$. By letting $m \rightarrow \infty$ in inequality (3.35), we know that $C=C^{\prime}$. This completes the proof.

Theorem 3.3. If

$$
\begin{aligned}
\lim _{m \rightarrow \infty} T_{j=1}^{\infty}\left[T\left(\rho_{0, k^{m+j-1} x}\left(k^{j+2 m} t\right), \rho_{0,-k^{m+j-1} x}\left(k^{j+2 m} t\right)\right)\right] \\
=1 \\
=\lim _{m \rightarrow \infty} T_{j=1}^{\infty}\left[T\left(\rho_{0, k^{m+j-1} x}\left(\frac{2 k^{2 j+3 m}}{k^{2}(k-1)} t\right), \rho_{k^{m+j-1} x, 0}\left(\frac{k^{2 j+3 m}\left(1-k^{2}\right)}{k^{2}(k-2)} t\right)\right),\right. \\
\left.\quad T\left(\rho_{0,-k^{m+j-1} x}\left(\frac{2 k^{2 j+3 m}}{k^{2}(k-1)} t\right), \rho_{-k^{m+j-1} x, 0}\left(\frac{k^{2 j+3 m}\left(1-k^{2}\right)}{k^{2}(k-2)} t\right)\right)\right]
\end{aligned}
$$

for all $x \in X$ and $t>0$ and

$$
\begin{aligned}
\lim _{m \rightarrow \infty} & T\left(\rho_{k^{m} x, k^{m} y}\left(k^{2 n-1} t\right), \rho_{k^{m} x, k^{m} y}\left(k^{2 n-1} t\right)\right) \\
& =1 \\
& =\lim _{m \rightarrow \infty} T\left(\rho_{k^{m} x, k^{m} y}\left(k^{3 n-1} t\right), \rho_{k^{m} x, k^{m} y}\left(k^{3 n-1} t\right)\right), \quad \forall x, y \in X, t>0,
\end{aligned}
$$

then there exist a unique quadratic mapping $Q: X \rightarrow Y$ and a unique cubic mapping $C: X \rightarrow Y$ such that 


$$
\begin{aligned}
& \mu_{f(x)-C(x)-Q(x)}(t) \\
& \geq T\left[T_{j=1}^{\infty}\left[T\left(\rho_{0, k^{j-1} x}\left(\frac{k^{j}}{2} t\right), \rho_{0,-k^{j-1}} x\left(\frac{k^{j}}{2} t\right)\right)\right],\right. \\
& T_{j=1}^{\infty}\left[T\left(\rho_{0, k^{j-1} x}\left(\frac{k^{2 j}}{k^{2}(k-1)} t\right), \rho_{k^{j-1} x, 0}\left(\frac{k^{2 j}\left(1-k^{2}\right)}{2 k^{2}(k-2)} t\right)\right),\right. \\
& \left.T\left(\rho_{0,-k^{j-1} x}\left(\frac{k^{2 j}}{k^{2}(k-1)} t\right), \rho_{-k^{j-1} x, 0}\left(\frac{k^{2 j}\left(1-k^{2}\right)}{2 k^{2}(k-2)} t\right)\right)\right], \quad \forall x \in X, t>0 .
\end{aligned}
$$

Proof. If $f_{e}(x)=(1 / 2)[f(x)+f(-x)]$ for all $x \in X$. Then $f_{e}(0)=0, f_{e}(-x)=f_{e}(x)$, and

$$
\begin{aligned}
& \mu_{f_{e}(x+k y)+f_{e}(x-k y)-k^{2}\left[f_{e}(x+y)+f_{e}(x-y)\right]-\left(2\left(k^{2}-1\right) / k^{2}(k-2)\right) f_{e}(k x)+\left(\left(k^{3}-k^{2}-k+1\right) / 2(k-2)\right) f_{e}(2 x)-\tilde{f}_{e}(2 y)+8 \tilde{f}_{e}(y)}(t) \\
& \quad \geq T\left(\rho_{x, y}(2 t), \rho_{-x,-y}(2 t)\right) \\
& \quad \geq T\left(\rho_{x, y}(t), \rho_{-x,-y}(t)\right), \quad \forall x, y \in X, t>0
\end{aligned}
$$

where $\tilde{f}_{e}(y):=f_{e}(y)-f_{e}(-y)$ for all $x, y \in X$. Hence, in view of Theorem 3.1, there exists a unique quadratic function $Q: X \rightarrow Y$ such that

$$
\mu_{Q(x)-f_{e}(x)}(t) \geq T_{j=1}^{\infty}\left[T\left(\rho_{0, k^{j-1} x}\left(k^{j} t\right), \rho_{0,-k^{j-1} x}\left(k^{j} t\right)\right)\right], \quad \forall x \in X, t>0 .
$$

Let $f_{o}(x)=(1 / 2)[f(x)-f(-x)]$ for all $x \in X$. Then $f_{o}(0)=0, f_{o}(-x)=-f_{o}(x)$ and

$$
\begin{aligned}
& \mu_{f_{o}(x+k y)+f_{o}(x-k y)-k^{2}\left[f_{o}(x+y)+f_{o}(x-y)\right]-\left(2\left(k^{2}-1\right) / k^{2}(k-2)\right) f_{o}(k x)+\left(\left(k^{3}-k^{2}-k+1\right) / 2(k-2)\right) f_{o}(2 x)-\tilde{f}_{o}(2 y)+8 \tilde{f}_{o}(y)}(t) \\
& \quad \geq T\left(\rho_{x, y}(2 t), \rho_{-x,-y}(2 t)\right) \\
& \quad \geq T\left(\rho_{x, y}(t), \rho_{-x,-y}(t)\right), \quad \forall x, y \in X, t>0,
\end{aligned}
$$

where $\tilde{f}_{o}(y):=f_{o}(y)-f_{o}(-y)$ for all $x, y \in X$. From Theorem 3.2, it follows that there exists a unique cubic mapping $C: X \rightarrow Y$ such that

$$
\begin{aligned}
& \mu_{C(x)-f_{o}(x)}(t) \geq T_{j=1}^{\infty}[ T\left(\rho_{0, k^{j-1} x}\left(\frac{2 k^{2 j}}{k^{2}(k-1)} t\right), \rho_{k^{j-1} x, 0}\left(\frac{k^{2 j}\left(1-k^{2}\right)}{k^{2}(k-2)} t\right)\right), \\
&\left.T\left(\rho_{0,-k^{j-1} x}\left(\frac{2 k^{2 j}}{k^{2}(k-1)} t\right), \rho_{-k^{j-1} x, 0}\left(\frac{k^{2 j}\left(1-k^{2}\right)}{k^{2}(k-2)} t\right)\right)\right], \quad \forall x \in X, t>0 .
\end{aligned}
$$

Obviously, (3.38) follows from (3.40) and (3.42). This completes the proof. 


\section{Acknowledgment}

This paper was supported by the Korea Research Foundation Grant funded by the Korean Government (KRF-2008-313-C00050).

\section{References}

[1] S. M. Ulam, Problems in Modern Mathematics, chapter 6, John Wiley \& Sons, New York, NY, USA, 1964.

[2] D. H. Hyers, "On the stability of the linear functional equation," Proceedings of the National Academy of Sciences of the United States of America, vol. 27, pp. 222-224, 1941.

[3] T. M. Rassias, "On the stability of the linear mapping in Banach spaces," Proceedings of the American Mathematical Society, vol. 72, no. 2, pp. 297-300, 1978.

[4] Z. Gajda, "On stability of additive mappings," International Journal of Mathematics and Mathematical Sciences, vol. 14, no. 3, pp. 431-434, 1991.

[5] J. Aczél and J. Dhombres, Functional Equations in Several Variables, vol. 31 of Encyclopedia of Mathematics and its Applications, Cambridge University Press, Cambridge, UK, 1989.

[6] T. Aoki, "On the stability of the linear transformation in Banach spaces," Journal of the Mathematical Society of Japan, vol. 2, pp. 64-66, 1950.

[7] D. G. Bourgin, "Classes of transformations and bordering transformations," Bulletin of the American Mathematical Society, vol. 57, pp. 223-237, 1951.

[8] M. E. Gordji, S. Kaboli Gharetapeh, E. Rashidi, T. Karimi, and M. Aghaei, “Ternary Jordan derivations in $C^{*}$-ternary algebras," Journal of Computational Analysis and Applications, vol. 12, no. 2, pp. 463-470, 2010.

[9] P. Găvruţa, "A generalization of the Hyers-Ulam-Rassias stability of approximately additive mappings," Journal of Mathematical Analysis and Applications, vol. 184, no. 3, pp. 431-436, 1994.

[10] P. Găvruta and L. Găvruta, "A new method for the generalized Hyers-Ulam-Rassias stability," International Journal of Nonlinear Analysis and Applications, vol. 1, no. 2, pp. 11-18, 2010.

[11] D. H. Hyers, G. Isac, and T. M. Rassias, Stability of Functional Equations in Several Variables, vol. 34 of Progress in Nonlinear Differential Equations and Their Applications, Birkhäuser Boston, Boston, Mass, USA, 1998.

[12] G. Isac and T. M. Rassias, "On the Hyers-Ulam stability of $\psi$-additive mappings," Journal of Approximation Theory, vol. 72, no. 2, pp. 131-137, 1993

[13] H. Khodaei and Th. M. Rassias, "Approximately generalized additive functions in several variables," International Journal of Nonlinear Analysis and Applications, vol. 1, pp. 22-41, 2010.

[14] C. Park and A. Najati, "Generalized additive functional inequalities in Banach algebras," International Journal of Nonlinear Analysis and Applications, vol. 1, pp. 54-62, 2010.

[15] C. Park and M. E. Gordji, "Comment on approximate ternary Jordan derivations on Banach ternary algebras [ Bavand Savadkouhi et al. J. Math. Phys. 50, 042303 (2009)]," Journal of Mathematical Physics, vol. 51, Article ID 044102, 7 pages, 2010.

[16] T. M. Rassias, "On the stability of functional equations and a problem of Ulam," Acta Applicandae Mathematicae, vol. 62, no. 1, pp. 23-130, 2000.

[17] T. M. Rassias, "On the stability of functional equations in Banach spaces," Journal of Mathematical Analysis and Applications, vol. 251, no. 1, pp. 264-284, 2000.

[18] P. Kannappan, "Quadratic functional equation and inner product spaces," Results in Mathematics. Resultate der Mathematik, vol. 27, no. 3-4, pp. 368-372, 1995.

[19] F. Skof, "Local properties and approximation of operators," Rendiconti del Seminario Matematico e Fisico di Milano, vol. 53, pp. 113-129, 1983.

[20] P. W. Cholewa, "Remarks on the stability of functional equations," Aequationes Mathematicae, vol. 27, no. 1-2, pp. 76-86, 1984.

[21] St. Czerwik, "On the stability of the quadratic mapping in normed spaces," Abhandlungen aus dem Mathematischen Seminar der Universität Hamburg, vol. 62, pp. 59-64, 1992.

[22] A. Grabiec, "The generalized Hyers-Ulam stability of a class of functional equations," Publicationes Mathematicae Debrecen, vol. 48, no. 3-4, pp. 217-235, 1996.

[23] K.-W. Jun and H.-M. Kim, "The generalized Hyers-Ulam-Rassias stability of a cubic functional equation," Journal of Mathematical Analysis and Applications, vol. 274, no. 2, pp. 267-278, 2002.

[24] S.-S. Chang, Y. J. Cho, and S. M. Kang, Nonlinear Operator Theory in Probabilistic Metric Spaces, Nova Science Publishers, Huntington, NY, USA, 2001. 
[25] M. E. Gordji, A. Ebadian, and S. Zolfaghari, "Stability of a functional equation deriving from cubic and quartic functions," Abstract and Applied Analysis, vol. 2008, Article ID 801904, 17 pages, 2008.

[26] M. E. Gordji and H. Khodaei, "Solution and stability of generalized mixed type cubic, quadratic and additive functional equation in quasi-Banach spaces," Nonlinear Analysis, vol. 71, no. 11, pp. 5629$5643,2009$.

[27] B. Schweizer and A. Sklar, Probabilistic Metric Spaces, North-Holland Series in Probability and Applied Mathematics, North-Holland Publishing, New York, NY, USA, 1983.

[28] A. N. Sherstnev, "On the notion of a random normed space," Doklady Akademii Nauk SSSR, vol. 149, pp. 280-283, 1963 (Russian).

[29] O. Hadžić and E. Pap, Fixed Point Theory in Probabilistic Metric Spaces, vol. 536 of Mathematics and Its Applications, Kluwer Academic, Dordrecht, The Netherlands, 2001.

[30] O. Hadžić, E. Pap, and M. Budinčević, "Countable extension of triangular norms and their applications to the fixed point theory in probabilistic metric spaces," Kybernetika, vol. 38, no. 3, pp. 363-382, 2002.

[31] E. Baktash, Y. J. Cho, M. Jalili, R. Saadati, and S. M. Vaezpour, “On the stability of cubic mappings and quadratic mappings in random normed spaces," Journal of Inequalities and Applications, vol. 2008, Article ID 902187, 11 pages, 2008.

[32] M. E. Gordji, M. B. Ghaemi, and H. Majani, "Generalized Hyers-Ulam-Rassias theorem in Menger probabilistic normed spaces," Discrete Dynamics in Nature and Society, vol. 2010, Article ID 162371, 11 pages, 2010.

[33] H. Khodaei and M. Kamyar, "Fuzzy approximately additive mappings," International Journal of Nonlinear Analysis and Applications, vol. 1, pp. 44-53, 2010.

[34] M. Mohamadi, Y. J. Cho, C. Park, F. Vetro, and R. Saadati, "Random stability on an additive-quadraticquartic functional equation," Journal of Inequalities and Applications, vol. 2010, Article ID 754210, 18 pages, 2010.

[35] D. Miheț, R. Saadati, and S. M. Vaezpour, "The stability of the quartic functional equation in random normed spaces," Acta Applicandae Mathematicae, vol. 110, no. 2, pp. 797-803, 2010.

[36] D. Miheț, R. Saadati, and S. M. Vaezpour, "The stability of an additive functional equation in Menger probabilistic $\varphi$-normed spaces," Mathematics, Slovak. In press.

[37] C. Park, "Fuzzy stability of an additive-quadratic-quartic functional equation," Journal of Inequalities and Applications, vol. 2010, Article ID 253040, 22 pages, 2010.

[38] C. Park, "Fuzzy stability of additive functional inequalities with the fixed point alternative," Journal of Inequalities and Applications, vol. 2009, Article ID 410576, 17 pages, 2009.

[39] C. Park, "A fixed point approach to the fuzzy stability of an additive-quadratic-cubic functional equation," Fixed Point Theory and Applications, vol. 2009, Article ID 918785, 24 pages, 2009.

[40] R. Saadati, S. M. Vaezpour, and Y. J. Cho, "Erratum: a note to paper "On the stability of cubic mappings and quartic mappings in random normed spaces"," Journal of Inequalities and Applications, vol. 2009, Article ID 214530, 6 pages, 2009.

[41] S. Shakeri, R. Saadati, and C. Park, "Stability of the quadratic functional equation in non-Archimedean L-fuzzy normed spaces," International Journal of Nonlinear Analysis and Applications, vol. 1, pp. 72-83, 2010.

[42] S.-S. Zhang, J. M. Rassias, and R. Saadati, "Stability of a cubic functional equation in intuitionistic random normed spaces," Applied Mathematics and Mechanics. English Edition, vol. 31, no. 1, pp. 21-26, 2010. 